\title{
Hypothesis derivation and its verification by a wholly automated many-objective evolutionary optimization system
}

\author{
Kazuhisa Chiba $^{1}$ (D) $\cdot$ Masataka Sawahara $^{1} \cdot$ Tsuyoshi Sumimoto $^{1} \cdot$ Taiki Hatta $^{1} \cdot$ Masahiro Kanazaki $^{2}$
}

Received: 15 April 2020 / Accepted: 5 February 2021 / Published online: 6 March 2021

(C) The Author(s) 2021

\begin{abstract}
This study has constructed a fully automated multidisciplinary and many-objective evolutionary design optimization system independent of computer environments to evaluate objective functions; the research applied it to a geometric design problem of a flyback booster for next-generation space transportation. In optimization involving objective functions to appraise the aero-/structural-dynamic performance with high fidelity, spatial discretization hinders the overall automation. This research has facilitated an efficient optimal design by wholly automating high-fidelity assessments, which designers had to implement manually, and has accomplished optimizations that directly contribute to real-world design problems. Moreover, this study would accumulate design knowledge for space transportation that the market is reviving. The total automated system yielded the embedding of geometric trait lines to ensure the discretization even for large curvature surfaces; the system innovated a robust automatic error-checking mechanism in the system's preprocess. Consequently, the entirely automatized optimization procured nondominated solution sets for more precise data analyses in a pragmatic execution period. Design informatics, a framework combining optimization and data analysis, functioned usefully in realworld design on flyback-booster geometry by materializing smooth deriving and verifying a design hypothesis; eventually, the research gained a new design principle.
\end{abstract}

Keywords Entire automatization system · Multidisciplinary and many-objective evolutionary optimization . Real-world pragmatic design · Computational fluid dynamics with unstructured mesh methods $\cdot$ Hypothesis derivation and its verification

\section{Introduction}

Design informatics (DI) is a methodology for efficiently coping with real-world design problems; DI composes three perspectives: problem definition, optimization, and data analysis [5]. There are numerous methods for optimization as the second viewpoint. However, multiobjective evolutionary computations (ECs) that exhaustively explore objectivefunction space must be proper ways for facilitating the

Kazuhisa Chiba

kazchiba@uec.ac.jp

1 The University of Electro-Communications, 1-5-1, Chofugaoka, Chofu, Tokyo 182-8585, Japan

2 Tokyo Metropolitan University, 6-6, Asahigaoka, Hino, Tokyo 191-0065, Japan significance of data analysis as the third viewpoint of DI [52]. Surrogate models, such as the radial basis function [4] and the Kriging model [42], which are remarkably attracting attention in recent years [31], have the substantial benefit of handling uncertainties [40]. Nevertheless, the number of individuals that assure the model's accuracy boosts with the number of design variables [47]. Since they are still approximate models, ECs are more reasonable alternatives for large-scale problems with enormous numbers of objective functions and design variables [36].

Designers should assess objective functions with highfidelity modes based on physics to gain results worthy of use in practical problems [43]. However, past studies have often shunned such problem definitions and have chosen simple evaluations such as mathematical modeling or estimation from a database due to system complexity and 
computational cost [2]. In aerospace vehicles, aerodynamics stipulates outer geometries of bodies; structural dynamics assures their feasibility; problem definitions cannot withdraw their design requirements generally. Although the problem with a simple geometry already accomplished full automation [38], spatial discretization for intricate geometries is a bottleneck for an entirely automated optimization covering aerodynamic/structural performances [34]; no research has resolved this issue. Any researchers desire to optimize efficiently regardless of geometric complexity and that optimizations directly contribute to designing real-world objects.

High-frequency and price reduction of space transportation are urgent priorities by the recent growth in small satellites' demands. Under this circumstance, reusable launch systems (RLSs) are one of the realizability in several aspects to attain the purposes as a successor of the Space Shuttle. Hence, studies have recently activated reusable space transportation, mainly in China [53], Russia [32], the E.U. [46], and the USA [16]. Meanwhile, Japan is currently conducting collaborative research on a two-stage RLS among some universities. Launch experiments of a prototype model are underway to establish a control law for launching an actual body [12]. The project is designing its trajectory and geometry simultaneously; the authors are studying the conceptual design on an RLS [48].

This study creates a wholly automated multidisciplinary and many-objective evolutionary optimization system for large-scale problems independent of computer surroundings to evaluate objective functions. The research would apply it to designing a flyback booster to amass design knowledge next-generation space transportation whose demand revives.

This treatise is composed as follows. Section 2 explains related researches on automated design optimizations and the present entirely automated optimization system. Section 3 expresses the definition of the real-world problem to which the study applies the present approach; also, the paper concisely outlines the utilized optimizers and datamining techniques. Section 4 reviews the application results. Section 5 verifies the obtained design hypothesis by investigating fluid physical mechanisms. Section 6 concludes this article.

\section{Wholly automated multiobjective evolutionary design optimization system}

Previous studies regarding automated preprocess had some trial and error: to automate the definition of geometries using parts of the CATIA ${ }^{\mathrm{TM}}$ package [9] and the generation of surface meshes from stereolithography data $(* . s t 1)$ to create 3D geometries [13]. The study by a structured mesh method on these extensions already earned an automated process for wing-fuselage geometry [44]. However, no research has still accomplished complete automatizations in unstructured mesh methods for highly expressive geometry representation.

The authors implemented the design optimizations so far (on a flyback booster [6], a regional jet airplane [7], and a supersonic demonstrator [5]) which was the challenge of automatizing the whole flow in the preprocess. Hence, there is no choice but to intervene in some human hands. Thus, an optimum aerodynamic design is an inefficient work that must be tougher (because designers have to perform the task that inevitably involves any people's hands for all individuals made by ECs). Automated execution of all processes for evolutionary design optimizations, including preprocess to post-process of computational fluid dynamics (CFD) analysis, is one of the goals of researchers who perform optimum aerodynamic design utilizing ECs for this way efficiently.

The following outlines the completely automated design optimization system constructed in this study. The essence of the entire automatization is in the following three points:

1. Embedding shape feature lines: since the system draws only one sideline, the next challenge is to improve the degree of freedom on making feature lines.

2. Automatic inspection in preprocess in CFD: the system implements an existence inspection of all the output files.

3. Executing CFD analysis on external computers:

(a) Check for communication errors using exit codes of ssh and scp commands.

(b) Eliminating dependency on external computer environments: a system should be ready to enable the use with minimum software preparation like the following:

(i) Job management system: Grid Engine, Slurm, and Workload Manager.

(ii) Commands required for shell script execution (for instance, bash, send, and greg).

(iii) A CFD solver.

Since system construction on LinuxOS is ordinary, to install a CFD solver is only crucial.

The rest portion will describe the details of the system.

The system adopts Eclipse ${ }^{1}$. developed by IBM $^{\circledR}$ in the integrated development surrounding (it is only a development environment, so it does not affect any program

\footnotetext{
1 "Eclipse Foundation" available online at https://www.eclipse.org/ [retrieved 5 Dec. 2018]
} 
executions); it constructs an optimization system using the MOEA Framework. ${ }^{2}$. It provides available ECs as opensource Java libraries. The following will proceed with the story with the content in line with this research's problem definition, assuming aerodynamic performance evaluation.

As shown in Fig. 1, when the system is activated, the MOEA Framework commences generating individuals. If an individual conflicts geometric constraints, the MOEA Framework repeats the creation until it obtains individuals that satisfy them for the population size. Then, it activates the parallel processing system (red boxes in Fig. 1); it processes each individual in parallel. The contents of this process are as follows:

(i) the preprocess for assessing objective functions

(ii) analyzing flow field by CFD

(iii) the post-process for calculating objectives' value

The system makes a folder /G\#_ $i$ / with generation number \# and personal identification number $i$ to evaluate in standalone for each individual.

What is vital for efficiently running the system is computing environments that perform CFD analyses. It constitutes an integrated development surrounding the terminal at hand, but CFD analyses drive the job to appropriate computers. This study employed the computers in the laboratory (Intel Xeon E5-26xx series: 9 nodes 156 cores, the parallel number is twice the number of cores) and the integrated supercomputation system AFI-NITY. ${ }^{3}$.

As described below, this research performed three optimizations. The present study has accomplished a time saving by achieving complete automation. However, 3rd optimization took roughly eight hours for one generation, even with the latest computing environment. The 1 st and 2nd optimizations, which served as trial calculations for the 30 generations, took about 20 days each; the 3rd optimization by a more sophisticated system took around 50 days for the 150 generations. Although the proposed approach and methodology have no limitations, they possess a temporal limit due to the time necessitated evaluating objective functions.

This study uses a surface/volume mesh generator MEGG3D (which involves the technical realms: surface triangulation with stereolithography data [21], surface triangulation with computer-aided design data [22], hybrid volume meshing [23], reliable isotropic tetrahedral meshing [25], multiple marching direction for hybrid mesh [29], hexahedral meshing [26], automatic local re-meshing [19],

\footnotetext{
2 "MOEA Framework" available online at http://moeaframework. org/ [retrieved 5 Dec. 2018]

3 "Advance Fluid Information Research Center, Institute of Fluid Science, Tohoku University" available online at http://www.ifs. tohoku.ac.jp/ afirc/afirc_eng/supercomputer/index.html [retrieved 19 Mar. 2020].
}

adaptive meshing [24], efficient hybrid surface and volume meshing [20], automatic local re-meshing for plural-type hybrid meshes [28], and hybrid meshing with an embedded surface [27]) and a CFD solver FaSTAR [17] developed by Japan Aerospace Exploration Agency to solve the compressible Navier-Stokes equations for each condition defined in the objective functions. A code based on HASA [15] generated by National Aeronautics and Space Administration for the Space Shuttle is modified to coordinate hybrid wing bodies; it estimates the empty structural weight.

\subsection{Preprocess}

For an aerodynamic analysis that adequately captures fluid phenomena, a geometry has to be defined accurately, discretize the surface of its airframe appropriately (surface mesh generation), spatially discretize the flow field suitably (volume mesh generation). The preprocess, including these works, is the most obstacle portion for automatization. The system delivers the following description according to the preprocess flowchart exhibited in Fig. 2.

\subsubsection{Generating point sequence of geometry surface}

The system stocks $\mathrm{dv}_{-} j$.txt with the information regarding all of the defined design variables; a geometry generator makes an input file geom_info_ $j$. set to form a point sequence data for a body surface. Then, it generates a point sequence structurally arranged in the direction of $x, y$, and $z$ with the name $j . \mathrm{p} 3 \mathrm{~d}$ to express the outline of a body surface. Finally, the system converts the $j$.p3d's format to an arrangement for a provisional unstructured surface mesh and stocks $j$. uns with it.

\subsubsection{Creating unstructured surface/volume meshes}

The system generates a ridge point sequence for boundaries at each surface region (zone) described in $j$. uns. It makes an unstructured surface mesh together with the $j$.uns information (in other words, the system prepares the part where it produces the ridgeline for zone borders). In this way, the system automatically creates the symmetric plane and the outer boundary surface; the computational space shuts. After that, the system forms an unstructured volume mesh using this surface mesh. To precisely capture the boundary layer, the volume mesh involves prism layers (1st layer of $y^{+} \sim 1$, at least 41 layers are laminated) in the vicinity of the wall surface-the data of a volume mesh retain within $j$.unv. Finally, the system converts $j$.unv to $j$. fsgrid in conjunction with the solver format. 


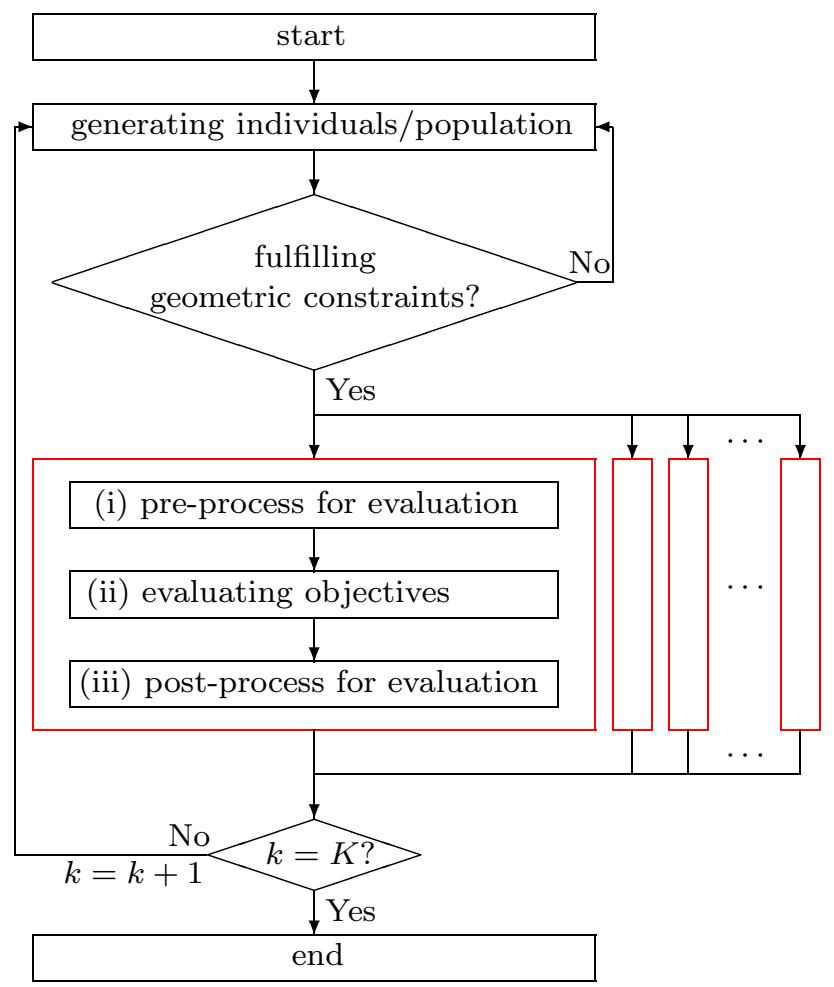

Fig. 1 Flowchart of the MOEA Framework. $k$ denotes the present generation number; it inputs $1,2, \ldots . K$ describes the upper limit of generation number in an EC

\subsubsection{Parameterizing body characteristics}

The system makes files (PARAMDAT $j$ and $\operatorname{SPDAT}_{-} j$ ) of geometric feature data (projected area, span length, body surface area, body volume, and center of gravity position) required for CFD analyses and post-process.

\subsection{Post-process}

Post-process has fewer intricacies in automation than the preprocess and already accomplished in our past researches, so there are no noteworthy points. As shown in Fig. 3, the system first combines a computational result divided on parallel computers into $j$.rslt. The system assesses each objective function using $j$.rslt and creates objs_pop_j $j$.txt, which describes objective-function values. The MOEA Framework reads objs_pop_j . txt of all the individuals in the population and (1) finishes if it reaches the stipulated number of generations or (2) generates individuals of the next generation if it does not attain.

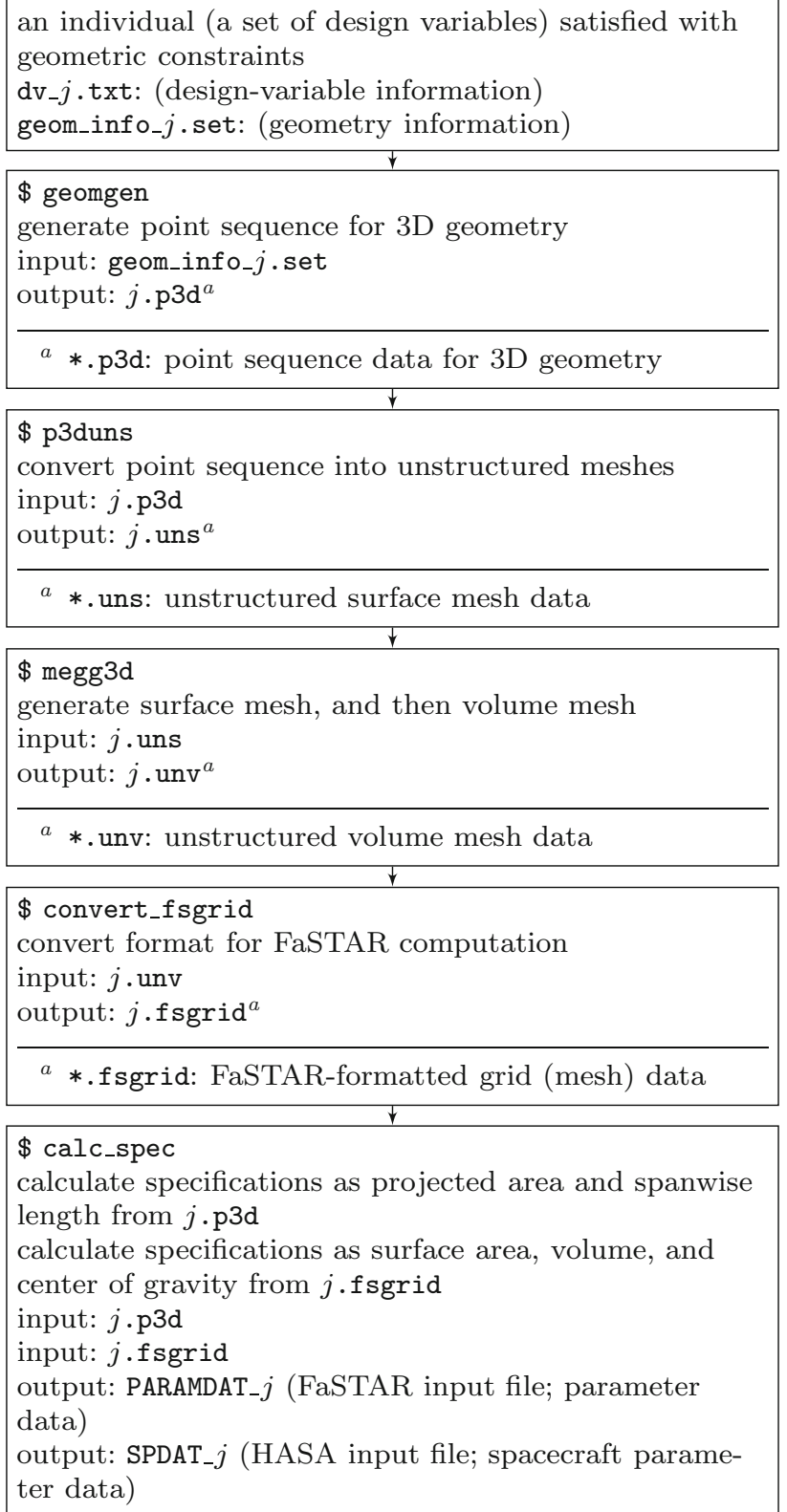

Fig. 2 Flowchart of the preprocess for CFD analyses. $j$ denotes the serial number of each individual in an EC, which the paper names with generation number \# and the individual number $i$ in a generation

\section{Application of the system to a real-world problem}

At present, several Japanese universities promote research on RLSs; one of the universities develops a fully reusable launch vehicle, WIRES [12]. The project is attempting to create a flyback booster for next-generation two-stage space transportation based on the optimized trajectory of WIRES. The optimal path fundamentally varies with the change in geometry. However, it is challenging to produce the database of aerodynamic performances required for 


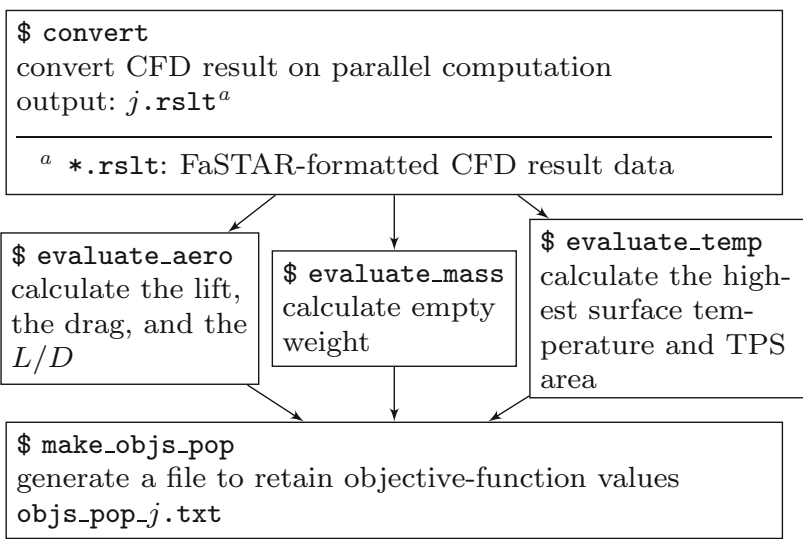

Fig. 3 Flowchart of the post-process for calculating objectivefunction values. $L / D$ denotes lift-to-drag ratio, and TPS is the abbreviation of thermal protection systems

trajectory optimization for each individual that a geometry optimization handles, so this is an issue for the future.

The trajectory considered in this study supposes an injection of $10 \mathrm{t}$ payload from the Tanegashima Space Center into a circular orbit at an altitude of $350 \mathrm{~km}$. The present optimization problem defined three objectives on aerodynamic performance: transonic, supersonic, and hypersonic speeds based on the trajectory. A booster should earn ranges as long as possible under the transonic and supersonic conditions for fly back to the launch site. A body should take a sequence with heaving the altitude to secure the range margin at the hypersonic design point where a booster and an orbiter separate. In an extension of aerodynamic performance, the problem determined the other three objective functions for structural weight and aerodynamic heating.

\subsection{Problem definition}

This problem defines six objective functions: three for aerodynamics (maximizing the lift-to-drag ratio $L / D$ at the Mach number $M=0.65,2.3$, and 6.8), one for the structures (minimizing empty weight), and two for aerodynamic heating (minimizing highest body surface temperature and

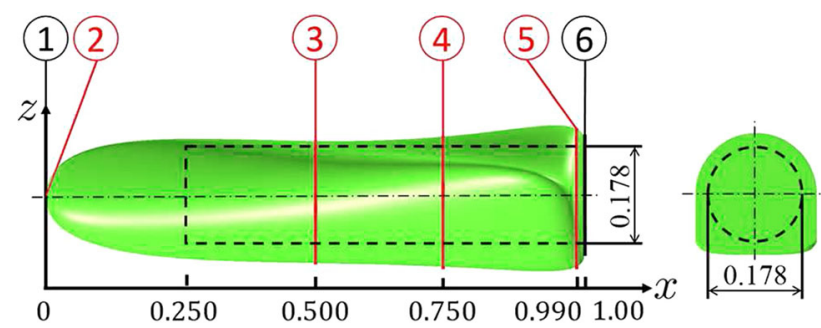

Fig. 4 Left: cross-sectional positions, right: tail surface. The dotted line represents a fuel tank with a fixed geometry body surface area where thermal protection system (TPS) attaches).

As shown in Fig. 4, the system generates six crosssectional shapes for the $x$-axis direction. The treatise utilizes section numbers (1) and (6) to satisfy later-described constraint conditions; the cross-sectional shape change in optimization is four locations with (2) to (5). Although the definition differs only in the (2) cross-section, the one cross-section uses ten design variables, as shown in Fig. 5, so the total design variables are 40 . Table 1 explains the definition of each design variable.

The problem defines constraints on the geometry; no regulation provides for the objective functions. The population size supposes to be ten. Considering the parallelization efficiency of computers that use the time to evaluate a single individual, the research adopted a strategy to suppress the population size and grow the number of generations.

\subsection{Optimizer}

Since one of the information desired by the multiobjective design optimization is the executable structure of the objective function space, the system chose ECs as metaheuristic optimizers, which perform global searches. Although many beneficial ECs endure, this study adopted two ECs. One is the strength Pareto evolutionary algorithm 2 (SPEA2) [54] for the 1st [8, 48] and the 2nd [18] optimizations. The other is a parallelized [37] multiobjective evolutionary algorithm based on decomposition (MOEA/ D) [51] for the 3rd optimization to gain nondominated solutions comprehensively.

Much research proposes new algorithms for metaheuristics; many methods have undergone various transitions and created the current state of affairs. Hence, this paper would not impart an exhaustive review of them here. However, reference [14] comprehensively assesses both single- and multi-objective algorithms and proffers a reliable overview of the current metaheuristics state.

Real-world problems typically hold multiple design requirements, i.e., numerous objective functions; they often prefer multi-objective algorithms. The current research topic is how to handle constraints [1] and deal with the growing number of objective functions. The latter problem, the many-objective problem [39], causes an extreme deterioration of convergence. Thus, decomposition-based methods are the most prevalent ones [49], but they abandon the exhaustive search design variable space.

To evaluate the performance of fluid phenomena generally solving the Navier-Stokes (NS) equation, the Millennium prize problems contain the NS equation; no one has yet proven existing its analytical solution. The flow field that the NS equation represents is complex and 
Fig. 5 Relationship among design variables $V_{n}$ and control points $\mathrm{P}_{m}$

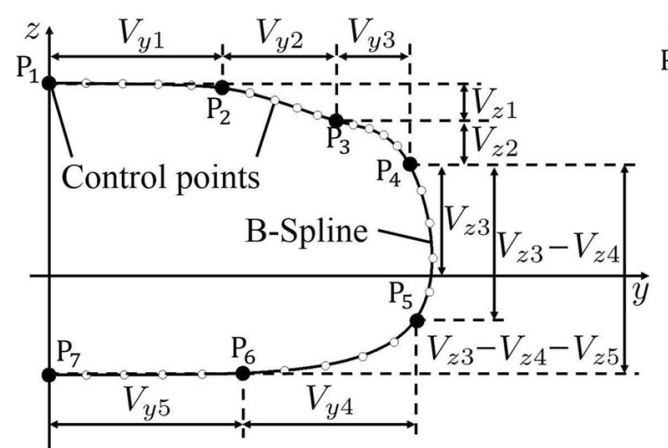

(a) at (2)

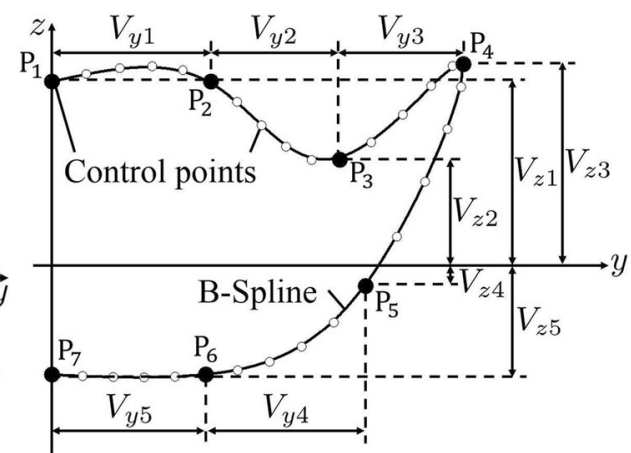

(b) from (3) to (5)

Table 1 Definition of design variables

\begin{tabular}{|c|c|c|c|c|c|}
\hline \multirow[t]{2}{*}{ Section number } & \multirow[t]{2}{*}{ Design variable $\mathrm{S} / \mathrm{N}$ number } & \multirow[t]{2}{*}{ Parameter sense } & \multirow[t]{2}{*}{ Symbol (refer to Fig. 5) } & \multicolumn{2}{|c|}{ Applicable range $[-]$} \\
\hline & & & & Lower & Upper \\
\hline \multirow[t]{2}{*}{ (2) } & $1,3,5,7,9$ & $y$-direction increment & $V_{y 1}, V_{y 2}, V_{y 3}, V_{y 4}, V_{y 5}$ & 0 & 0.073 \\
\hline & $2,4,6,8,10$ & $z$-direction increment & $V_{z 1}, V_{z 2}, V_{z 3}, V_{z 4}, V_{z 5}$ & 0 & 0.087 \\
\hline \multirow[t]{4}{*}{ (3) } & $11,13,15,17,19$ & $y$-direction increment & $V_{y 1}, V_{y 2}, V_{y 3}, V_{y 4}, V_{y 5}$ & 0 & 0.073 \\
\hline & 12 & $z$-direction increment & $V_{z 1}$ & 0 & 0.218 \\
\hline & $14,16,18$ & & $V_{z 2}, V_{z 3}, V_{z 4}$ & @@0.218 & 0.218 \\
\hline & 20 & & $V_{z 5}$ & @@0.218 & 0 \\
\hline \multirow[t]{4}{*}{ (4) } & $21,23,25,27,29$ & $y$-direction increment & $V_{y 1}, V_{y 2}, V_{y 3}, V_{y 4}, V_{y 5}$ & 0 & 0.35 \\
\hline & 22 & $z$-direction increment & $V_{z 1}$ & 0 & 0.218 \\
\hline & $24,26,28$ & & $V_{z 2}, V_{z 3}, V_{z 4}$ & @@0.218 & 0.218 \\
\hline & 30 & & $V_{z 5}$ & @@0.218 & 0 \\
\hline \multirow[t]{4}{*}{ (5) } & $31,33,35,37,39$ & $y$-direction increment & $V_{y 1}, V_{y 2}, V_{y 3}, V_{y 4}, V_{y 5}$ & 0 & 0.35 \\
\hline & 32 & & $V_{z 1}$ & 0 & 0.218 \\
\hline & $34,36,38$ & & $V_{z 2}, V_{z 3}, V_{z 4}$ & @@0.218 & 0.218 \\
\hline & 40 & & $V_{z 5}$ & @@0.218 & 0 \\
\hline
\end{tabular}

strongly nonlinear, calculating gradient information extremely difficult. Optimization of airplane aerodynamic geometry by the adjoint method, a mathematical optimization approach using gradient information of objective functions, has been attained [35]. Still, it is incapable of adopting this study, where the airframe geometry transforms drastically.

Meanwhile, the engineering realm often employs surrogate models, not to mention ECs, because it requires no gradient information of objective functions [33]. However, to guarantee the precise response surface, it is vital to prepare initial samples five to ten times larger than the number of design variables [47], which is also unsuitable for the present study with many design variables.

\subsection{Data-mining techniques}

A scatter plot matrix (SPM) is one of the oldest, most straightforward, but most adaptable and accessible scatter plots [11]. SPM visualizes a multidimensional dataset by allocating data axes to graphical axes and describing data samples as points in Cartesian space stipulated by the axes. The traits of SPM visualization are to be able to use 3D graphics as well as color, shape, and size of points. This study utilizes iSPM [50].

Functional analysis of variance (ANOVA) quantifies the contribution rates to the variance of a model [30]. ANOVA decomposes the model's total variance into that of each design variable and their interactions to estimate their impact quantitatively. Furthermore, there are various techniques for visualizing information as sturdy devices for knowledge discovery [41]. The filtering techniques, including triangular matrix representations (TMR) [3], 
have proven to be serviceable for tasks where visual representations can find relationships, clusters, outliers, gaps, and other patterns [45].

\section{Hypothesis derivation via optimization and data-mining results}

This section describes consecutive flow to derive a design hypothesis for a target vehicle using the design informatics approach. Essential knowledge for practical designers is the latent design information in a high-dimensional problem space composed of objective functions and design variables. The combination of optimization and data analysis is useful for the discovery and is an inseparable complementary relation.

\subsection{Review of the results of two optimizations}

This subsection concisely reviews the knowledge that the optimizations already indicated, which is crucial for interpreting the data-mining results. The system used the identical optimization algorithm (SPEA2) for the 1st and the 2nd optimizations, with the same problem definitions except for the ditch-related design variables, with the equivalent generation, identical initial population, and the same computing environment.

\subsubsection{Knowledge from the optimization results}

A ditch should bring a positive effect in the lift and a negative influence on the drag. As a result of the optimizations shown in Fig. 6, the research anticipates that a ditch will positively impact the low-speed $L / D$. Therefore, this study focuses on improving the $L / D$ at $M=0.65$; it does not discuss how to grow the $L / D$ at $M=6.8$.

Difference of design strategies: optimal geometries of each objective function for the $L / D$ explicate the following diversity in design strategies.

- $L / D$ at $M=0.65$ grows by raising the lift.

- $L / D$ at $M=6.8$ rises by reducing the drag. The optimization makes bodies slender to decline the drag in the hypersonic range. Since slim bodies concomitantly enable to diminish the surface temperature, they simultaneously improve three of the six objective functions; it must stipulate the evolutionary trend.

- The strategy for raising the $L / D$ at $M=2.3$ is related to that for the $L / D$ at $M=6.8$, but with scope for gaining the lift.

Influence of ditch:

- Positive effect on the lift: since a ditch facilitates vortex form, it grows vortex lift.

- Negative influence on the drag: the induced drag rises due to the vortex lift; the friction drag first increases with the expansion of the wetted area.

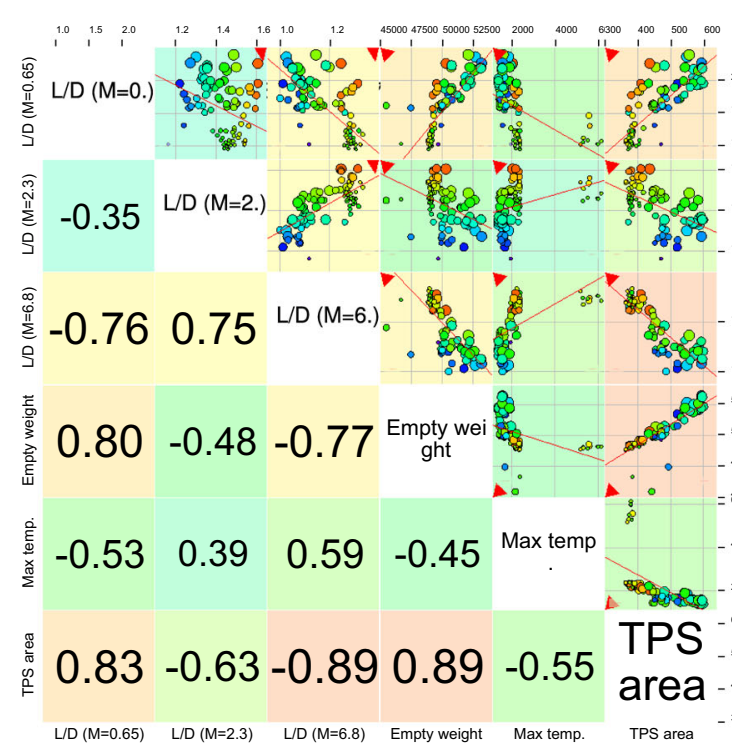

(a) the 1 st result considering ditch (103 individuals).

Fig. 6 SPM of nondominated solutions for 30 generations by SPEA2 in the objective-function space. The size/color of the plots reflects the values of the $L / D$ at $M=0.65 / M=2.3$. Red lines/triangles signify

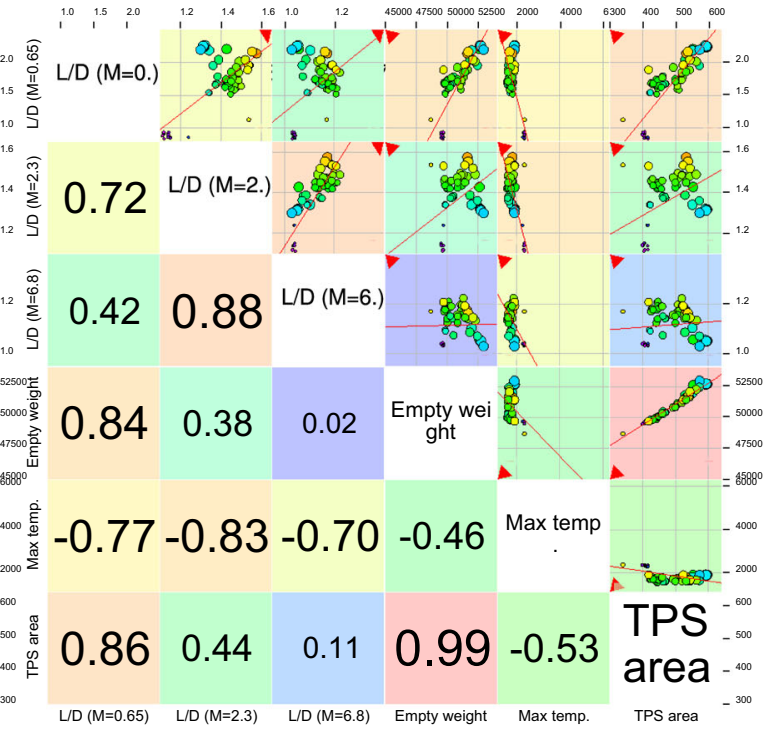

(b) the 2 nd result without considering ditch (81 individuals).

regression lines/optimum directions. Numbers on lower triangular matrices specify correlation coefficients 


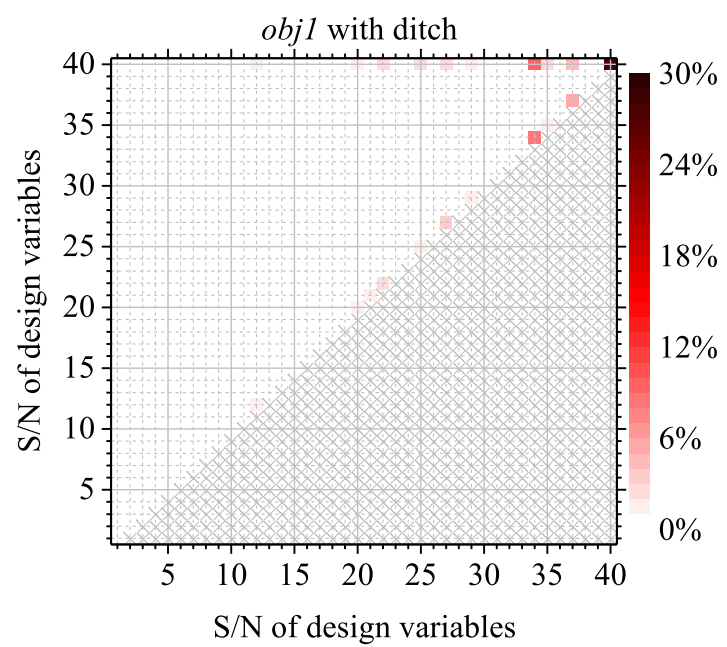

(a)

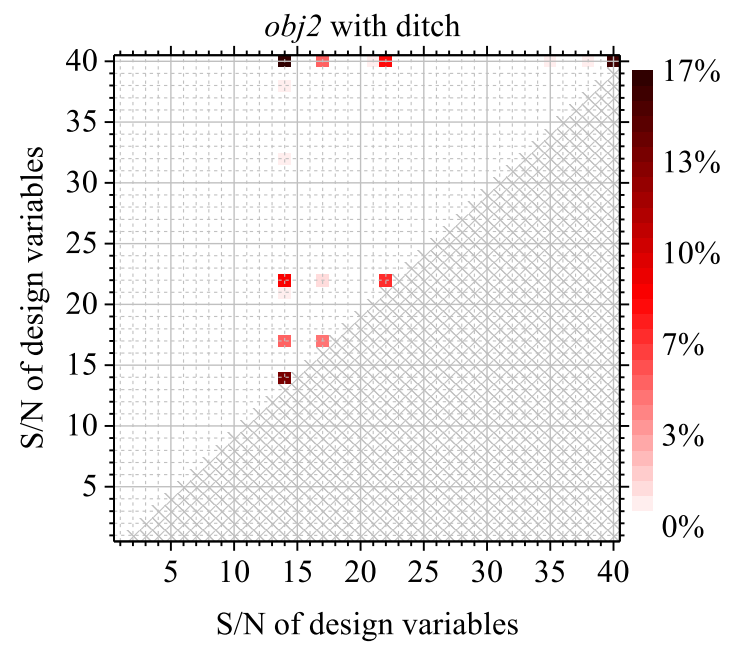

(b)

Fig. 7 TMR of ANOVA results for the dataset of the 1st optimization, which accidentally deals with ditches, for $\mathbf{a}$ the $L / D$ at $M=0.65$ and $\mathbf{b}$ the $L / D$ at $M=2.3$

\subsubsection{Design information from scatter plot matrix}

The 1st and the 2nd optimization results shown in Fig. 6 present the effect of the presence or absence of a ditch on the transonic/supersonic $L / D$ as follows:

- To eliminate a ditch deteriorates the $L / D$ at $M=0.65$, approximately $5.4 \%$.

- To delete a ditch worsens the $L / D$ at $M=2.3$, approximately $1.9 \%$.

- The $L / D$ at $M=6.8$ also declines, but it links to the $L / D$ at $M=2.3$.

Initially, to gain the $L / D$ at $M=0.65$ is more reliant on the lift than that at $M=2.3$, so to add a ditch must produce a more effect on gaining the lift than an impact on increasing the drag.

\subsection{Results by data mining}

Data mining aims to verify whether the accidentally created ditch on the back of a booster contributes to improving the $L / D$. So, data-mining results have to prove the following two perspectives: (1) the dataset from the 1st optimization that considers a ditch affects design variables involved in ditch generation, (2) the dataset from the 2 nd optimization that excludes a ditch does not impact on design variables required for ditch representation. Note that the ANOVA results for each objective function quantify each design variable's contribution, assuming that the total influence on an objective function is $100 \%$. The present ANOVA results cannot quantitatively discuss the relative effects among objective functions.

\subsubsection{Result for dataset with ditch}

$L / D$ at $M=0.65:$ TMR in Fig. 7 a indicates that the design variables with substantial influence are $d v 34, d v 37$, and $d v 40$; the design variables with weak effect are $d v 12, d v 20$, $d v 22$, and $d v 27$. The beneficial design variables register that the design strategies for improving the $L / D$ at $M=$ 0.65 are (a) spreading the body in the spanwise direction and making the bottom as flat as possible, and (b) providing a ditch. To prolong the wetted area, to stretch the body in the spanwise direction is the most valuable for raising the lift, which affects improving the $L / D$ at $M=0.65$. Therefore, the design variable's influence $(d v 20, d v 27, d v 37$, $d v 40$ ) for expanding a body in the spanwise direction and flattening the bottom surface is relatively more massive than the design variables $(d v 12, d v 22, d v 34)$ for providing a ditch. However, the provision of a ditch is undoubtedly useful in improving the $L / D$.

1. $\quad d v 34\left(V_{z 2}^{\varpi}\right)^{4}$ : to raise the difference from $V_{z 1}$ boosts the curvature of the B-Spline curve from $\mathrm{P}_{1}$ to $\mathrm{P}_{3}$, resulting in a deeper ditch; the ditch affects the low-speed $L / D$. Note that $V_{z 1}$ tends to be significant to serve the constraint of securing the tank volume, so $V_{z 2}$ inclines to be small.

2. $\quad d v 37\left(V_{y 4}^{(5)}\right)$ decreasing this value widens the flat bottom region in the spanwise direction; growing the pressure on the bottom of a body gains the lift.

3. $d v 40\left(V_{z 5}^{(5)}\right)$ : by reducing the bottom thickness and flattening body in the $z$-direction, the base matures a

\footnotetext{
${ }^{4}$ For example, $d v 34$ symbolizes the design variable $V_{z 2}$ of the (5) cross-section displayed in Table 1 and Fig. 5, so this paper expresses it as $V_{z 2}^{(5)}$.
} 
flat geometry. The identical design concept as $d v 37$ contributes to lift repair.

$L / D$ at $M=2.3$ : TMR in Fig. $7 \mathrm{~b}$ indicates that the design variables with substantial influence are $d v 14, d v 17, d v 22$, and $d v 40$. Since drag alleviation helps to improve the $L / D$ at $M=2.3$, unlike optimizing the $L / D$ at $M=0.65$, no geometry widens horizontally and tends to be elongated. Hence, the impact of the design variables $(d v 17, d v 40)$ that expand the body horizontally and the design variables $(d v 14, d v 22)$ that supplement a ditch to the lift is relatively robust in the latter.

\subsubsection{Result for dataset without ditch}

$L / D$ at $M=0.65$ : since all design variables indicated by TMR shown in Fig. 8a are irrelevant to a ditch, it does not affect on the $L / D$. The abilities of the design variables that have direct and indirect effects are abstracted below.

- design variables with direct effects:

1. $d v 10: V_{z 5}^{2}$ has the effect of gaining the lift by making the bottom of a body nose as flat as possible.

2. $d v 35: V_{y 3}^{\curvearrowleft}$ extends the area near a body tail in the spanwise direction and creates a stabilizer to enhance the lift.

- design variables with indirect effects:

1. $\quad d v 11, d v 23: V_{y 1}^{(3}$ and $V_{y 2}^{(4)}$ widen a body sideways and gain the lift.

2. $d v 40: V_{z 5}^{(5)}$ raises the lift by flattening the bottom near a body tail.

$L / D$ at $M=2.3$ : as all design variables indicated by TMR shown in Fig. 8b are independent of a ditch, the impact of a ditch faded away in the $L / D$ at $M=2.3$ as well as in the $L /$ $D$ at $M=0.65$. The capabilities of design variables that affect the $L / D$ at $M=2.3$, as shown by TMR, are as follows.

- design variables with direct influences:

1. $d v 10: V_{z 5}^{(2)}$ is a variable that ultimately transforms the $L / D$ because it is useful in raising the lift by flattening the bottom of a body nose and in diminishing the drag by making the curvature.

- design variables with indirect effects:

1. $d v 8, d v 20, d v 40: V_{z 4}^{(2)}, V_{z 5}^{(3)}$, and $V_{z 5}^{(5)}$ have effects comparable to $d v 10$.

2. $d v 22\left(V_{z 1}^{\oplus}\right)$ : the direct effect is weak; the result is more robust when combined with $d v 10$, which implies that the cross-sectional shape transformation in the flow direction affects the $L / D$. In the supersonic range, the distribution of the crosssectional area of a body in the flow direction generally desires alteration monotonically from the sonic boom theory [10]. The combination of $d v 10$ and $d v 22$ must deviate from a monotonic increase, leading to a rise in the wave drag, resulting in the $L /$ $D$ deterioration. In particular, since $d v 22$ represents the (4) cross-section, its cross-section is likely to disturb area distribution because there commences forming a stabilizer. In any case, $d v 22$ does not affect the lift.

\section{Hypothesis verification}

Since the data mining inferred the correctness of the design hypothesis in the previous section, this section will declare the fluid physical mechanism of the theory that a ditch on the back of a booster body raises the $L / D$ in the transonic speed. The two optimizations mentioned in Sect. 4 have insufficient individuals because the system suspended the optimizations without abundant evolution, partly because this research implemented them as the 1st and the 2nd trials of the system. These two experiments revealed that the system could execute regardless of computational environments used to evaluate objective functions. Hence, the 3rd fully automated optimization using a parallelized MOEA/D performed again for three months. As a result of evolving 152 generations with a population of ten individuals, the optimization procured 407 nondominated solutions. The 3rd optimization supposes under the identical condition as the 2nd optimization, filling any ditch on a body back.

Figure 9 visualizes the obtained nondominated solutions by SPM in the objective-function space. Compared to Fig. 6, the 3rd optimization more widely distributes nondominated solutions; SPM represents the surface shape of them more appropriately. The following will add a ditch to the best/worst three individuals with the $L / D$ at $M=0.65$ extracted from the result; the treatise would scrutinize the fluid physical mechanism that a ditch effects on the $L / D$ growth.

Figure 10 compiles the $L / D$ change rates, the lift, and the drag when the system modification deliberately appends a ditch to the six respective individuals. This figure indicates the rest information:

- The aerodynamic performances of the three best geometries deteriorate in the opposite direction. Since the best three bodies already improve the $L / D$ at $M=$ 0.65 via the optimization, a ditch reduces the $L /$ 


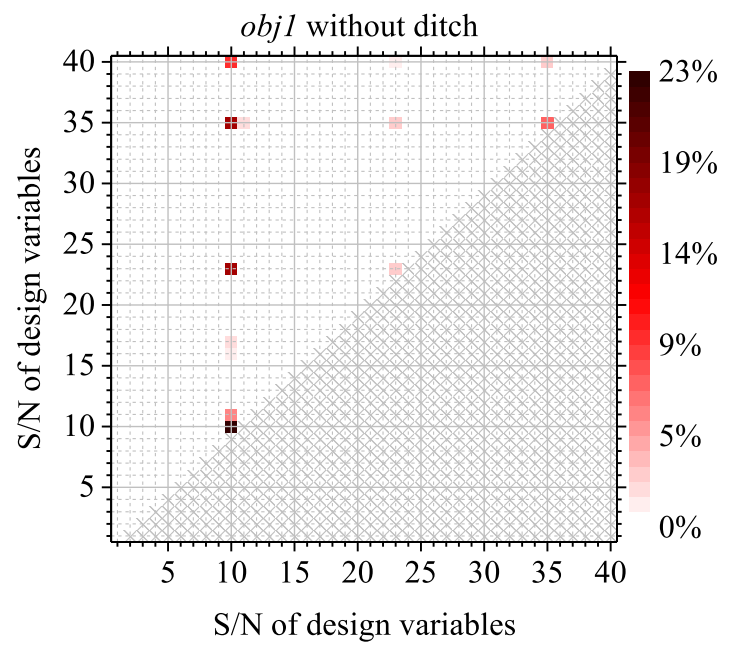

(a)

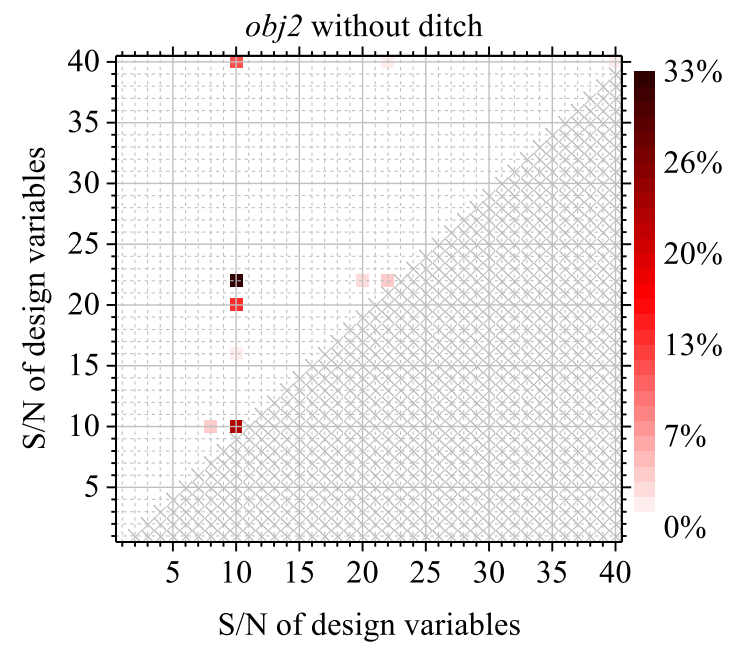

(b)

Fig. 8 TMR of ANOVA results for the dataset of the 2nd optimization, which solves the inverse problem to eliminate a ditch, for a the $L / D$ at $M=0.65$ and $\mathbf{b}$ the $L / D$ at $M=2.3$

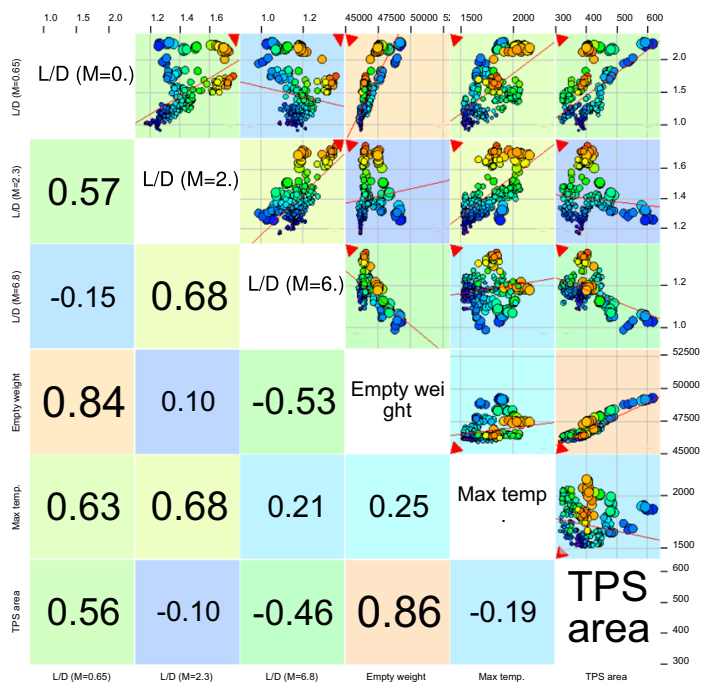

Fig. 9 SPM of 407 nondominated solutions for 152 generations by a parallelized MOEA/D in the objective-function space. The size/color of the plots reflects the values of the $L / D$ at $M=0.65 / M=2.3$. Red lines and red triangles signify regression lines and optimum directions, respectively. Numbers on lower triangular matrices specify correlation coefficients (Color figure online)

$D$ adversely. The drag diminishes, but the lift also decreases, so the $L / D$ worsens eventually.

- In the case of three worst geometries, although the lift rises, the drag also grows, so there are two cases where the $L / D$ improves and deteriorates.

To add a ditch harms the $L / D$ performance for bodies that the $L / D$ at $M=0.65$ is optimized explicitly. Still, it improves for many nondominated solutions compromised concerning other objective functions. Besides, since the

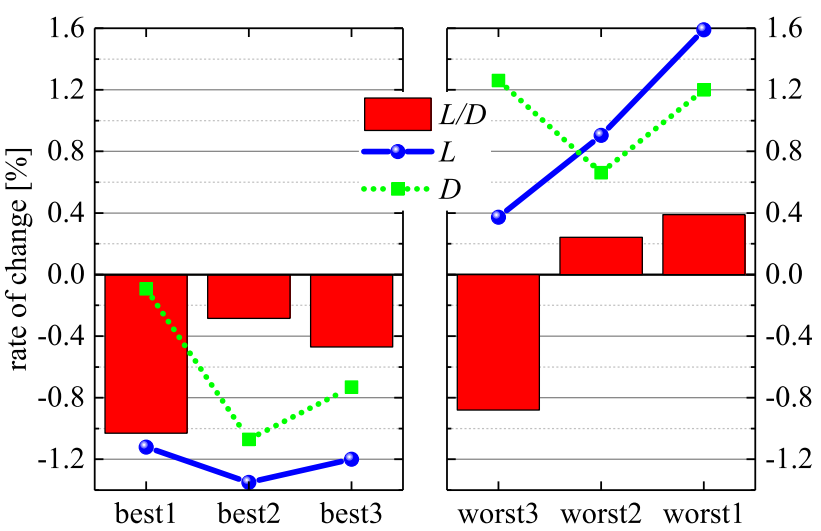

Fig. 10 Change rates in the $L / D$, the lift, and the drag for the three individuals with the best and the worst $L / D$ at $M=0.65$, respectively, when the system modification appended a ditch to each body intentionally

ditch shape joined this time is obscure, there is room to investigate an appropriate ditch design.

best $_{2} /$ worst $_{1}$ has the highest change rate in the lift among the best/worst three; Fig. 11 illustrates the distinction in the flow field with and without a ditch. In this way, the study scrutinizes the fluid physical mechanism that a ditch effects on the lift. In best 2 , adding a ditch decelerates the flow near itself and extends the high-pressure field to the rear of the body, which directly participates in diminishing in the lift. Conversely, worst $_{1}$ attributes the lift growth to the event that the ditch's addition accelerated the flow near itself and shifted the high-pressure region to the front of the body. This case appended the ditch irresponsibly, but if a ditch is duly made at the back of a body to accelerate the flow, it would assuredly enhance the lift. 


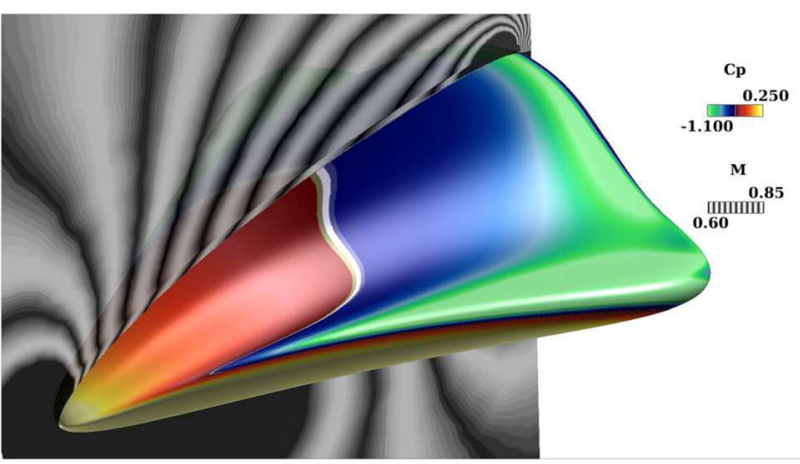

(a)

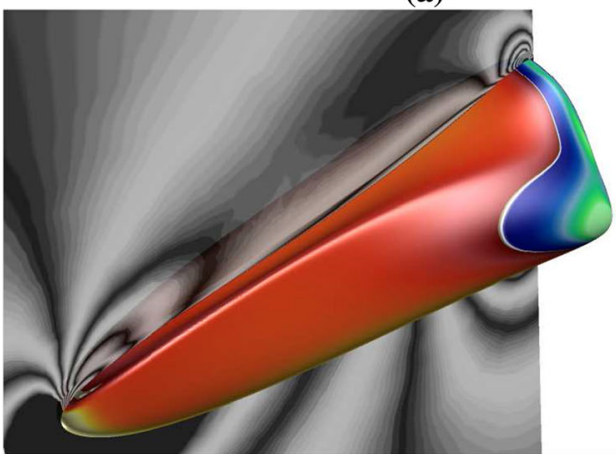

(c)

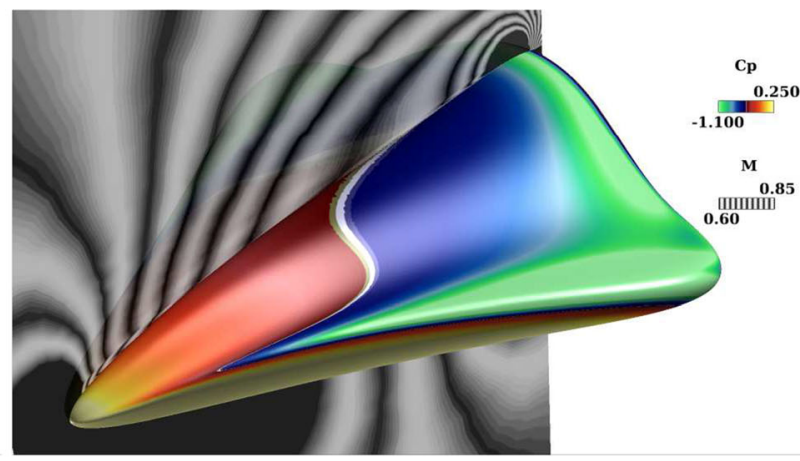

(b)

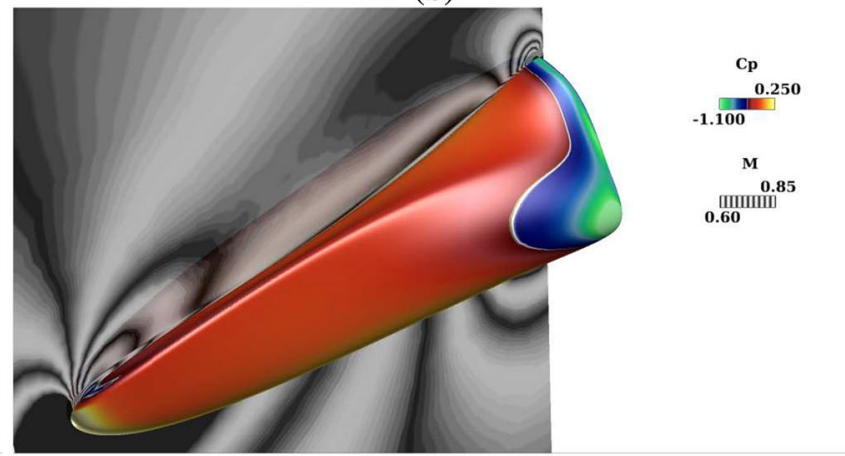

(d)

Fig. 11 Distinction of CFD results with/without a ditch. a best $t_{2}$ without a ditch, $\mathbf{b}$ best $_{2}$ with a ditch, $\mathbf{c}$ worst $_{1}$ without a ditch, and $\mathbf{d}$ worst $_{1}$ with a ditch

\section{Conclusion}

This study has developed a wholly automated multidisciplinary and many-objective evolutionary design optimization system independent of computer surroundings to assess objective functions; the research applied it to the geometric design problem of a flyback booster for nextgeneration space transportation. No fully automated optimization with precisely evaluating aero-/structural-dynamic performance had accomplished for intricate geometries due to the bottleneck spatial discretization. Efficient optimizations should be imperative for direct contributions to the design of actual industrial products. Besides, designers must accumulate design principles for space transportation, which are in resurgent demand. The entire automation resulted from the system constructed by facilitating the discretization of surface topography by preparing geometric feature lines, providing a robust programmed error-checking mechanism in the preprocess to assess objective functions, and eliminating the dependence on computer environments.

Consequently, because the system improved the realworld design problem's efficiency using evolutionary computations, it brought nondominated-solution sets to the data analyses required for the post-process of optimizations in a pragmatic execution period. Likewise, the created system derived a new design hypothesis on the flybackbooster geometry because of gaining the quality of data analyses. Case studies based on the hypothesis verified the theory and gained a new design principle.

Acknowledgements JHPCN (jh180507), JSPS KAKENHI (JP19K12095), and the Collaborative Research Project of the Institute of Fluid Science, Tohoku University (J19I076) supported a part of the research. FieldView19.0 created whole CFD visual images, which FieldView CFD, Inc. provides via its University Partners Program.

\section{Declaration}

Conflict of interest The authors declare they have no conflict of interest.

Open Access This article is licensed under a Creative Commons Attribution 4.0 International License, which permits use, sharing, adaptation, distribution and reproduction in any medium or format, as long as you give appropriate credit to the original author(s) and the source, provide a link to the Creative Commons licence, and indicate if changes were made. The images or other third party material in this article are included in the article's Creative Commons licence, unless indicated otherwise in a credit line to the material. If material is not included in the article's Creative Commons licence and your intended use is not permitted by statutory regulation or exceeds the permitted use, you will need to obtain permission directly from the copyright holder. To view a copy of this licence, visit http://creativecommons. org/licenses/by/4.0/. 


\section{References}

1. Afshari H, Hare W, Tesfamariam S (2019) Constrained multiobjective optimization algorithms: review and comparison with application in reinforced concrete structures. Appl Soft Comput 83:1-18. https://doi.org/10.1016/j.asoc.2019.105631

2. Arias-Montano A, Coello CAC, Mezura-Montes E (2012) Multiobjective evolutionary algorithms in aeronautical and aerospace engineering. IEEE Trans Evol Comput 16(5):662-694. https:// doi.org/10.1109/TEVC.2011.2169968

3. Birkenmeier GF, Heatherly HE, Kim JY, Park JK (2000) Triangular matrix representations. J Algebra 230(2):558-595. https:// doi.org/10.1006/jabr. 2000.8328

4. Buhmann MD (2000) Radial basis functions. Acta Numer 9:1-38. https://doi.org/10.1017/S0962492900000015

5. Chiba K, Makino Y, Takatoya T (2012) Design-informatics approach for intimate configuration of silent supersonic technology demonstrator. J Aircr 49(5):1200-1211. https://doi.org/10. 2514/1.C031116

6. Chiba K, Obayashi S, Nakahashi K (2006) Design exploration of aerodynamic wing shape for reusable launch vehicle flyback booster. J Aircr 43(3):832-836. https://doi.org/10.2514/1.12782

7. Chiba K, Oyama A, Obayashi S, Nakahashi K, Morino H (2007) Multidisciplinary design optimization and data mining for transonic regional-jet wing. J Aircr 44(4):1100-1112. https://doi.org/ $10.2514 / 1.17549$

8. Chiba K, Sumimoto T, Sawahara M (2019) Completely automated system for evolutionary design optimization with unstructured computational fluid dynamics. In: Proceedings of international conference on intelligent systems, metaheuristics \& swarm intelligence. ACM. https://doi.org/10.1145/3325773. 3325778

9. Clarich A, Poloni C (2007) Multi-objective optimisation in modeFRONTIER for aeronautic applications. In: Evolutionary methods for design, optimization and control

10. Darden CM (1977) Sonic boom theory-its status in prediction and minimization. J Aircr 14:569-576. https://doi.org/10.2514/3. 58822

11. Elmqvist N, Dragicevic P, Fekete J (2008) Rolling the dice: multidimensional visual exploration using scatterplot matrix navigation. IEEE Trans Vis Comput Graph 14(6):1539-1548. https://doi.org/10.1109/TVCG.2008.153

12. Fujikawa T, Yonemoto K, Yamasaki H, Ichige M, Ura Y, Gossamsetti GS, Ohki T, Kubono Y, Shirakata K, Tsukamoto H, Matsuoka Y, Tobiyama H, Kamemoto E, Ichinomiya K, Moriyama D, Hirakida S, Sugimachi T, Faroux D, Morito T, Masuda I, Katou T (2017) Research and development of winged reusable rocket: current status of experimental vehicles and future plans. In: Proceedings on Asia-Pacific International Symposium on Aerospace Technology. JSASS, Soul, Republic of Korea

13. Hahn AS (2010) Vehicle Sketch Pad: parametric geometry for conceptual aircraft design. AIAA Paper 2010-0657

14. Halim AH, Ismail I, Das S (2021) Performance assessment of the metaheuristic optimization algorithms: an exhaustive review. Artif Intell Rev 54:1-87. https://doi.org/10.1007/s10462-02009906-6

15. Harloff GJ, Berkowitz BM (1988) HASA-hypersonic aerospace sizing analysis for the preliminary design of aerospace vehicles. NASA-CR-182226

16. Harris M (2019) The heavy lift: Blue Origin's next rocket engine could power our return to the moon. IEEE Spectr 56:26-30. https://doi.org/10.1016/j.asoc.2019.1056311

17. Hashimoto A, Murakami K, Aoyama T, Ishiko K, Hishida M, Sakashita M, Lahur PR (2012) Toward the fastest unstructured CFD code "FaSTAR". AIAA Paper 2012-1075
18. Hatta T, Sawahara M, Chiba K (2019) Many-objective multidisciplinary evolutionary design for hybrid-wing-body-type flyback booster on an entirely automated system. In: Proceedings on international conference on Evolutionary and Deterministic Methods for Design, Optimization, and Control with Applications to Industrial and Societal Problems 2019. ECCOMAS

19. Ito Y, Murayama M, Yamamoto K, Shih AM, Soni BK (2009) Efficient computational fluid dynamics evaluation of small device locations with automatic local remeshing. AIAA J 47(5):1270-1276. https://doi.org/10.2514/1.40875

20. Ito Y, Murayama M, Yamamoto K, Shih AM, Soni BK (2011) Efficient hybrid surface and volume mesh generation for viscous flow simulations. AIAA Paper 2011-3539

21. Ito Y, Nakahashi K (2002) Direct surface triangulation using stereolithography data. AIAA J 40(3):490-496. https://doi.org/10. $2514 / 2.1672$

22. Ito Y, Nakahashi K (2002) Surface triangulation for polygonal models based on CAD data. Int $\mathrm{J}$ Numer Methods Fluids 39(1):75-96. https://doi.org/10.1002/fld.281

23. Ito Y, Nakahashi K (2004) Improvements in the reliability and quality of unstructured hybrid mesh generation. Int $\mathrm{J}$ Numer Methods Fluids 45(1):79-108. https://doi.org/10.1002/fld.669

24. Ito Y, Shih AM, Koomullil RP, Kasmai N, Jankun-Kelly M, Thompson D (2009) Solution adaptive mesh generation using feature-aligned embedded surface meshes. AIAA J 47(8):1879-1888. https://doi.org/10.2514/1.39378

25. Ito Y, Shih AM, Soni BK (2004) Reliable isotropic tetrahedral mesh generation based on an advancing front method. In: Proceedings of the 13th International Meshing Roundtable. Williamsburg, VA, pp 95-105

26. Ito Y, Shih AM, Soni BK (2009) Octree-based reasonable-quality hexahedral mesh generation using a new set of refinement templates. Int J Numer Methods Eng 77(13):1809-1833. https://doi. org/10.1002/nme. 2470

27. Ito Y, Shih AM, Soni BK (2011) Hybrid mesh generation with embedded surfaces using a multiple marching direction approach. Int J Numer Methods Fluids 67(1):1-7. https://doi.org/10.1002/ fld. 1962

28. Ito Y, Shih AM, Soni BK (2011) Three dimensional automatic local remeshing for two or more hybrid meshes. Int $\mathrm{J}$ Numer Methods Fluids 66(12):1495-1505. https://doi.org/10.1002/fld. 2324

29. Ito Y, Shih AM, Soni BK, Nakahashi K (2007) Multiple marching direction approach to generate high quality hybrid meshes. AIAA J 45(1):162-167. https://doi.org/10.2514/1.23260

30. Jones DR, Schonlau M, Welch WJ (1998) Efficient global optimization of expensive black-box functions. J Glob Optim 13(4):455-492. https://doi.org/10.1023/A:1008306431147

31. Kanazaki M, Matsuno T, Maeda K, Kawazoe H (2015) Efficient global optimization applied to wind tunnel evaluation based optimization for improvement of flow control by plasma actuator. Eng Optim 47(9):1226-1242. https://doi.org/10.1080/0305215X. 2014.958733

32. Kolychev AV, Kernozhitskii VA, Chernyshov MV (2019) Thermionic methods of cooling for thermostressed elements of advanced reusable launch vehicles. Russ Aeronaut 62:669-674. https://doi.org/10.3103/S1068799819040184

33. Mack Y, Goel T, Shyy W, Haftka R (2007) Surrogate modelbased optimization framework: a case study in aerospace design. In: Studies in computational intelligence, vol 51. Springer, Berlin Heidelberg. https://doi.org/10.1007/978-3-540-49774-5_14

34. Martins JRRA, Alonso JJ, Reuther JJ (2004) High-fidelity aerostructural design optimization of a supersonic business jet. J Aircr 41(3):523-530. https://doi.org/10.2514/1.11478

35. Martins JRRA, Alonso JJ, Reuther JJ (2005) A coupled-adjoint sensitivity analysis method for high-fidelity aero-structural 
design. Optim Eng 6:33-62. https://doi.org/10.1023/B:OPTE. 0000048536.47956.62

36. Mukhopadhyay A, Maulik U, Bandyopadhyay S, Coello CAC (2014) A survey of multiobjective evolutionary algorithms for data mining: Part I. IEEE Trans Evol Comput 18(1):4-19. https:// doi.org/10.1109/TEVC.2011.21699686

37. Nebro AJ, Durillo JJ (2010) A study of the parallelization of the multi-objective metaheuristic MOEA/D. In: Learning and intelligent optimization, LNCS, vol 6073. Springer, Berlin Heidelberg, pp 303-317. https://doi.org/10.1007/978-3-642-13800-3_32

38. Obayashi S, Sasaki D, Takeguchi Y, Hirose N (2000) Multiobjective evolutionary computation for supersonic wing-shape optimization. IEEE Trans Evol Comput 4:182-187. https://doi. org/10.1109/TEVC.2011.21699687

39. Ramírez A, Romero JR, Ventura S (2019) A survey of manyobjective optimisation in search-based software engineering. J Syst Softw 149:382-395. https://doi.org/10.1109/TEVC.2011. 21699688

40. Rumpfkeil MP (2013) Optimizations under uncertainty using gradients, hessians, and surrogate models. AIAA J 51(2):444-451. https://doi.org/10.2514/1.J051847

41. Sacha D, Stoffel A, Stoffel F, Kwon BC, Ellis G, Keim DA (2014) Knowledge generation model for visual analytics. IEEE Trans Vis Comput Graph 20(12):1604-1613. https://doi.org/10. 1109/TVCG.2014.2346481

42. Sacks J, Welch W, Mitchell T, Wynn H (1989) Design and analysis of computer experiments. Stat Sci 4(4):409-423

43. Samareh JA (2001) Survey of shape parameterization techniques for high-fidelity multidisciplinary shape optimization. AIAA J 39(5):877-884. https://doi.org/10.2514/2.1391

44. Sasaki D, Yang G, Obayashi S (2004) Automated aerodynamic optimization system for SST wing-body configuration. Trans Jpn Soc Aeronaut Space Sci 46(154):230-237. https://doi.org/10. 2322/tjsass.46.230

45. Shneiderman B (2008) Extreme visualization: squeezing a billion records into a million pixels. In: Proceedings of the 2008 ACM SIGMOD international conference on management of data, pp 3-12. https://doi.org/10.1145/1376616.1376618
46. Simplício P, Marcos A, Bennani S (2019) Reusable launchers: development of a coupled flight mechanics, guidance, and control benchmark. J Spacecr Rockets 56:74-89. https://doi.org/10.2514/ 1.A34429

47. Simpson TW, Mauery TM, Korte JJ, Mistree F (2001) Kriging models for global approximation in simulation-based multidisciplinary design optimization. AIAA J 39(12):2233-2241. https:// doi.org/10.2514/2.1234

48. Sumimoto T, Chiba K, Kanazaki M, Fujikawa T, Yonemoto K, Hamada N (2019) Evolutionary multidisciplinary design optimization of blended-wing-body-type flyback booster. In: AIAA Paper 2019-0703 on the 57th AIAA Aerospace Science Meeting. AIAA

49. Tanabe R, Ishibuchi H (2020) A framework to handle multimodal multiobjective optimization in decomposition-based evolutionary algorithms. IEEE Trans Evol Comput 24(4):720-734. https://doi. org/10.1006/jabr.2000.83285

50. Tatsukawa T, Oyama A, Kohira T, Kemmotsu H, Miyachi H (2017) iSPM - an interactive scatterplot matrix for visualizing multidimensional engineering data. In: Proceedings of the IEEE Visualization Conference. IEEE, Phoenix, Arizona, USA

51. Zhang Q, Li H (2007) MOEA/D: a multiobjective evolutionary algorithm based on decomposition. IEEE Trans Evol Comput 11(6):712-731. https://doi.org/10.1109/TEVC.2007.892759

52. Zhao X, Wu Y, Cui W, Du X, Chen Y, Wang Y, Lee LD, Qu H (2018) SkyLens: visual analysis of skyline on multi-dimensional data. IEEE Trans Vis Comput Graph 24(1):246-255. https://doi. org/10.1109/TVCG.2017.2744738

53. Zhou H, Wang X, Cui N (2020) Glide guidance for reusable launch vehicles using analytical dynamics. Aerosp Sci Technol 98:1-2. https://doi.org/10.1006/jabr.2000.83288

54. Zitzler E, Laumanns M, Thiele L (2001) SPEA2: improving the strength Pareto evolutionary algorithm. TIK-Report No. 103, Computer Engineering and Communication Networks Lab., ETH Zurich

Publisher's Note Springer Nature remains neutral with regard to jurisdictional claims in published maps and institutional affiliations. 